\title{
CONDICIÓN AMBIENTAL DE LOS POBLADOS DE CERRILLOS, SABANA BONITA Y PEÑAS BLANCAS, PUNTARENAS, COSTA RICA
}

\author{
Environmental conditions of the communities Cerrillos, Sabana Bonita y Peñas \\ Blancas, Puntarenas, Costa Rica
}

María José Chassoul Acosta ${ }^{1}$

\author{
Recibido 5/III/2015
}

Aprobado $8 / \mathrm{VI} / 2015$

\section{Resumen}

Los resultados mostrados en este artículo son parte de una investigación más grande que propone un modelo de desarrollo sostenible para las comunidades de Cerrillos, Sabana Bonita y Peñas Blancas de Esparza, Puntarenas. Específicamente se analiza el equilibrio existente entre las actividades que realizan los habitantes y el uso que le brindan a los recursos naturales, a partir de los datos obtenidos de la aplicación de una encuesta que indaga sobre variables como: energía, suelo, agua, residuos, capacitación ambiental y prácticas de gestión ambiental utilizadas. Se incluye además una descripción de la conservación, recuperación y protección realizada y el riesgo y vulnerabilidad ambiental presente. Se detectó que estos poblados presentan diversos problemas ambientales como son: erosión y compactación de suelos por la ganadería, contaminación de nacientes por el mal manejo de estas, contaminación de quebradas por vertido de aguas residuales de casas y chancheras y mal manejo de residuos; así como problemas de deslizamiento en el poblado de Peñas Blancas. Sin embargo, a través de la Asociación pro cuenca del rio Jabonal se están realizando acciones para mejorar la situación ambiental actual.

\section{Palabras clave}

Condición ambiental, sub cuenca del río Jabonal, Cerrillos, Sabana Bonita, Peñas Blancas

\section{Abstract}

The results shown in this article are part of a further research. The research proposes a sustainable development model for the communities Cerrillos, Sabana Bonita and Peñas Blancas of Esparza, Puntarenas. There is an analysis of data obtained through a conducted survey that searches variables related to energy, soil, water, waste, environmental training and the use of environmental management systems. The analysis is supported by other investigations done in those communities. Additionally, there is an explanation of the environmental conservation, restoration and protection done and the environmental risk and vulnerability. The aim of this research was to know the balance among the activities done by the inhabitants and the use of natural resources. The environmental issues found in those communities are: soil erosion, soil compaction by 
livestock, pollution of rivers by sewage of houses, pig farmers and inappropriate waste management. Furthermore, there is a landslide in the community of Peñas Blancas. However, through the Association pro cuenca del río Jabonal there are some actions to improve the environmental issues.

\section{Key words}

Environmental conditions, sub cuenca del río Jabonal, Cerrillos, Sabana Bonita, Peñas Blancas

\section{Introducción}

Los poblados de Cerrillos, Sabana Bonita y Peñas Blancas, se ubican en la sub cuenca del río Jabonal perteneciente a la provincia de Puntarenas. Aproximadamente el $42 \%$ del área se utiliza para el desarrollo de actividades agropecuarias, con predominio de la producción ganadera. Dicha actividad sumada a la pobreza presente en la zona, han ocasionado prácticas inapropiadas de uso del suelo, del agua y de los ecosistemas asociados. Por lo que, con el propósito de conocer el impacto en el ambiente de estas prácticas, se realizó un diagnóstico de la condición ambiental de estos poblados. La cual se entiende como la situación de los recursos, como agua, suelo y cobertura vegetal, factores que determinan la capacidad productiva de los espacios y las condiciones de vida de las personas (Sepúlveda et al 2002).

Específicamente el diagnóstico se centró en el análisis de variables como energía, suelo, agua, residuos y capacitación ambiental. En energía se indagó sobre el consumo mensual promedio de electricidad por vivienda y el uso de fuentes alternativas para la generación de energía. El suelo se investigó a través del uso, la contaminación y degradación debido al manejo inadecuado de éste y la fertilidad. En agua se examinó la hidrografía de la zona, las fuentes de abastecimiento, la calidad y las fuentes de contaminación. No se realizó ningún estudio sobre calidad de agua debido a que investigaciones anteriores realizadas por Auquilla, Astorga y Jiménez en el año 2006 y PROGAI en el año 2007, ya lo habían efectuado. Con respecto a residuos, el análisis se basó en tres parámetros principales: la cantidad de residuos producidos por tipo (doméstico, agrícola y peligroso), la disposición final que le brindan y la tecnología empleada para el manejo de éstos. La capacitación ambiental se fundamentó en la cantidad de cursos en los que los habitantes han participado.

Además se incluye una descripción de la conservación, recuperación y protección realizada en la zona y el riesgo y vulnerabilidad ambiental presente. 


\section{Materiales y métodos}

\section{Área de estudio}

La investigación se realizó, entre los años 2011 y 2012, en la sub cuenca del río Jabonal, cuenca del río Barranca, provincia de Puntarenas, Costa Rica. Específicamente en los poblados de Cerrillos, Sabana Bonita y Peñas Blancas. Ver figura 1.

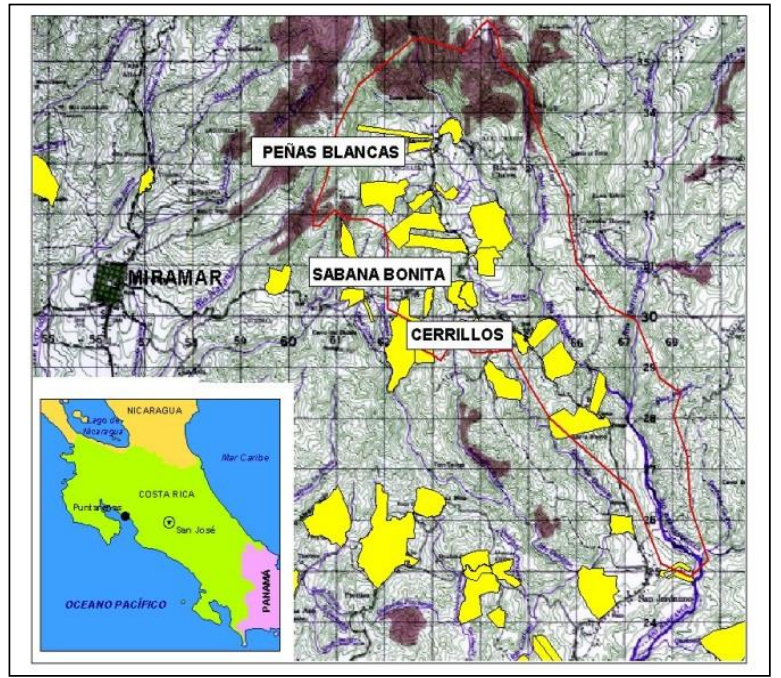

Fuente: UCR-PROGAI 2007

Figura 1. Ubicación de la sub cuenca del río Jabonal y los poblados de Cerrillos, Sabana Bonita y Peñas Blancas

\section{Instrumento}

Para recopilar la información se aplicó en las viviendas de los tres poblados un cuestionario conformado por 74 preguntas, de las cuales 22 se refieren específicamente a la condición ambiental. Estas preguntas brindan información sobre variables como energía, suelo, agua, residuos, capacitación ambiental y prácticas de gestión ambiental utilizadas. También se realizó la observación directa de las actividades.

\section{Aplicación}

En esta investigación no se utilizó muestra. Se trabajó con el 100\% de las viviendas de los tres poblados, pero se logró encuestar al 74\%. La encuesta se aplicó a 28 familias de Cerrillos, 14 de Sabana Bonita y 29 de Peñas Blancas, sumando un total de 71 familias encuestadas.

\section{Análisis de datos}

El análisis de datos se realizó por medio del software de análisis estadístico Minitab 16. Se aplicó tanto estadística descriptiva como estadística inferencial.

La prueba de Kruskal-Wallis se utilizó para comparar las tres poblaciones estudiadas con respecto a la variable analizada. Específicamente para determinar si existía diferencia de comportamiento 
entre poblados. La prueba de proporciones se utilizó con la finalidad de evaluar si los resultados obtenidos de diferentes variables presentaban la misma proporción en los tres poblados. En todas las pruebas se utilizó un nivel de significancia de 0,05.

\section{Resultados y discusión}

\section{Energía}

El 99\% (70) de las viviendas de los poblados analizados cuenta con servicio eléctrico. Este servicio es brindado por el Instituto Costarricense de Electricidad (ICE). Lo utilizan, en su mayoría, para el alumbrado de las casas y el uso de electrodomésticos. Para cocinar utilizan gas y leña. El gasto promedio en este rubro es de 10 mil colones por mes, lo cual indica que en estas casas existe un consumo menor a los $200 \mathrm{kWh}$. Este consumo es inferior al promedio de consumo para Esparza, el cual según el Atlas para el desarrollo humano cantonal de Costa Rica (PNUD, 2011), es de 785,7 kWh por cliente. Si se compara con el consumo de los clientes de Montes de Oca (1014,5 kWh), cantón con el mejor índice de desarrollo humano de Costa Rica, el consumo es 5 veces inferior.

Con respecto al uso de fuentes alternativas de producción de energía, únicamente el 3\% (2) de las viviendas encuestadas utilizan un biodigestor para la producción de gas. Estas familias utilizan el gas generado para cocinar, ahorrándose 10 mil colones por mes, aproximadamente. Según manifestaron los encuestados existe interés en la instalación de biodigestores, sin embargo no lo han construido debido a la inversión monetaria que deben realizar.

\section{Suelo}

- Uso de suelo

La sub cuenca de rio Jabonal, en la cual se ubican los poblados de Cerrillos, Sabana Bonita y Peñas Blancas, tiene un área, aproximada, de $43 \mathrm{~km}^{2}$. En esa sub cuenca el $33 \%$ del territorio lo utilizan para la ganadería. De acuerdo a Auquilla, Astorga y Jiménez (2006, 83), "los suelos se clasifican como inceptisoles, de textura franca a franca arcillosa, quebrados, fuertemente ondulados con zonas escarpadas y pendientes de 30 a $60 \%$ ".

El $42 \%$ del suelo de la sub cuenca se utiliza para fines agropecuarios, principalmente ganadería, y el $58 \%$ posee cobertura boscosa (Mende, citado por Villalobos y Ramírez, 2010). De acuerdo a lo manifestado por los entrevistados de los tres poblados estudiados, al uso agropecuario (ganadería y siembra de hortalizas, verduras y café), le dedican, aproximadamente, $6,5 \mathrm{~km}^{2}$ o 650 hectáreas y a la protección de bosque, aproximadamente, $2,2 \mathrm{~km}^{2}$ o 220 hectáreas. Es importante aclarar que estos datos, son un estimado de lo que los encuestados consideran que utilizan y protegen. 
- Contaminación y degradación

De acuerdo con una serie de talleres realizados por el Programa Institucional de Gestión Ambiental de la Universidad de Costa Rica - PROGAI, en el año 2007, uno de los principales problemas ambientales presentes en la sub cuenca del río Jabonal, es la pérdida de suelo productivo por causas de la erosión por viento y escorrentía (PROGAI, 2007). También Villalobos y Ramírez (2010), establecen que el suelo en la sub cuenca presenta problemas por sobrepastoreo, deforestación y, específicamente en Peñas Blancas, deslizamiento debido a falla.

De acuerdo con lo manifestado por los encuestados, aproximadamente, el $50 \%$ dice tener problemas de erosión en las fincas. Además consideran que las principales fuentes de contaminación del suelo son la aplicación de herbicidas y el vertido de boñiga. Contaminación que se podría disminuir y eliminar si se emplearan sistemas silvopastoriles. En estos sistemas la protección de los árboles y arbustos es muy importante debido a que éstos preservan el agua y el suelo, disminuyen la escorrentía y ayudan a la infiltración del agua. Además la cubierta vegetal en asociación con los bosques riparios puede prevenir deslizamientos de tierras (Auquilla, 2005). Para lograr la implantación de estos sistemas, los habitantes de la zona requieren ayuda externa, tanto en capacitación como en la identificación de posibles fuentes de financiamiento.

- Fertilidad del suelo

De acuerdo al mapa de Edafoaptitud, el cual toma en cuenta el potencial de uso agrícola por fertilidad del suelo y las categorías de uso de la tierra (Universidad Nacional y Municipalidad de Esparza, 2010), el terreno de la zona de estudio, en su mayoría, presenta un índice de alta fragilidad ambiental, indicador de un suelo de muy baja fertilidad. También se observan zonas, en menor medida, de moderada y baja fragilidad ambiental. Lo cual indica que son zonas de moderada y alta fertilidad.

De las tres comunidades, Peñas Blancas es la que muestra en al mapa muy baja fertilidad. Zona donde se presentan severos problemas de erosión, poca profundidad, que va de los 30 a $60 \mathrm{~cm}$, y moderada pedregosidad. Comprende áreas montañosas con pendientes por encima del $75 \%$. Son tierras con condiciones no aptas para el uso agrícola, por lo que se recomienda la conservación del bosque y en áreas con usos diferentes la regeneración natural (Universidad Nacional y Municipalidad de Esparza 2010). Según esta fuente, Cerrillos presenta al norte zonas de muy baja fertilidad y al sur zonas de moderada y alta fertilidad. La fertilidad moderada se debe a limitaciones por pendientes fuertemente onduladas, erosión severa, texturas en el suelo y subsuelo finas, lo cual indica un terreno de fertilidad moderada. En la zona de alta fertilidad se encuentran suelos profundos, ligeramente pedregosos, y buenas condiciones de drenaje. Sabana Bonita 
presenta zonas de muy baja fertilidad en la mayoría de su territorio y al oeste zonas de alta fertilidad, debido a la presencia de erosión moderada, pendiente ondulada y la textura.

De acuerdo con estos resultados se podría descartar la implementación de cualquier tipo de siembra en el poblado de Peñas Blancas, sin embargo, en conversaciones mantenidas con los señores Madrigal y Villalobos (comunicación personal, octubre 2012) ambos pobladores de Sabana Bonita, dicen que Peñas Blancas, por evidencia en los sembradíos realizados, presenta una mejor cosecha que en los poblados de Sabana Bonita y Cerrillos. Por este motivo, sería necesario realizar un estudio más profundo y detallado sobre la fertilidad de suelos de la zona. Un estudio interdisciplinario que tome en cuenta diversos factores que pueden afectar la fertilidad y no solo el tipo de suelo.

Agua

- Hidrografía

La sub cuenca del río Jabonal, pertenece a la Cuenca del Río Barranca en el Pacífico Central de Costa Rica. Se encuentra a 700 metros de altitud sobre el nivel del mar y tiene una precipitación anual de $3200 \mathrm{~mm}$ (Ibrahim, citado por Soto, 2011). Esta sub cuenca es irrigada por varias quebradas y específicamente en la zona de estudio, se ubican las quebradas Limonal, Estrellal, Beneficio, La Perra, Honda y el río Guatuso.

- Abastecimiento de agua

En Sabana Bonita, el 100\% de las casas se abastece con agua de naciente. En Cerrillos, el 61\% (17) de las viviendas se abastecen de agua del acueducto del A y A y el 39\% (11) se abastece con agua de naciente. En Peñas Blancas, todas las casas se abastecen con agua de naciente. En algunos casos estas nacientes se encuentran dentro de la misma finca y en otros el agua proviene de nacientes de fincas vecinas.

De acuerdo al Estado de la Nación (2011), el 78,5\% de la población ubicada en zonas rurales del país cuentan con provisión de agua por acueductos. Este valor es mucho mayor al encontrado en los poblados estudiados, donde únicamente el $24 \%$ de las viviendas cuentan con servicio de agua brindado por Acueductos y Alcantarillados. El 76\% restante se abastece de agua de naciente y desconoce la calidad del agua consumida. Sin embargo, que el $24 \%$ de la población reciba agua de un acueducto del A y A, no garantiza que consuman agua con calidad adecuada para consumo humano, debido a que de acuerdo a estudio realizado por Mora y Araya (2008), el 2,2\% del agua brindada por esta institución es no potable. Afirmación que se comprueba con los datos que se presenta a continuación. 
- Calidad del agua

El estudio realizado por el Programa de gestión ambiental integral (PROGAI) de la Universidad de Costa Rica en el año 2007, demuestra que el agua consumida en las viviendas ubicadas en la sub cuenca del río Jabonal, contiene coliformes. Para este estudio, se realizó un muestreo en 20 domicilios ubicados en San Jerónimo, Peñas Blancas, Cerillos, Sabana Bonita y Carrera Buena. Los resultados obtenidos muestran que en 16 de 20 casas $(80 \%)$ se detectaron coliformes totales (de 2 hasta $5000 \mathrm{NMP} / 100 \mathrm{ml}$ ) y en 12 de 20 viviendas (60\%), se detectaron coliformes fecales (de 2 hasta $1600 \mathrm{NMP} / 100 \mathrm{ml}$ ) (PROGAI 2007). El valor máximo permitido de coliformes totales para agua de consumo humano es menor a 20 NMP/100 (MINAE, 2007).

En estudio realizado por Auquilla, Astorga y Jiménez (2006) en 8 nacientes de la zona, en el cual analizaron la calidad del agua por medio de análisis físico-químicos y bacteriológicos, se muestra que los parámetros de calidad de agua que excedieron el nivel crítico para consumo humano fueron la demanda química de oxígeno (DQO), los sólidos suspendidos (SS), el fósforo total y los coliformes fecales. En el cuadro 1, se muestra el valor obtenido por parámetro y el máximo permitido por el Reglamento para la evaluación y clasificación de la calidad de cuerpos de agua superficiales (MINAE 2007).

Cuadro 1. Parámetros físico-químicos y microbiológicos promedios obtenidos en 8 nacientes ubicadas en la sub cuenca del río Jabonal, Esparza, Puntarenas

\begin{tabular}{|l|l|l|}
\hline Parámetro & Valor obtenido & Valor permitido \\
\hline DQO & $19,7 \mathrm{mg} / \mathrm{l}$ & $<20 \mathrm{mg} / \mathrm{l}$ \\
\hline Sólidos suspendidos & $14,07 \mathrm{mg} / \mathrm{l}$ & $<10 \mathrm{mg} / \mathrm{l}$ \\
\hline Fósforo total & $0,13 \mathrm{mg} / \mathrm{l}$ & $0,1 \mathrm{mg} / \mathrm{l}$ \\
\hline Coliformes fecales & $330(\mathrm{NMP} / 100 \mathrm{ml})$ & $<20 \mathrm{NMP} / 100$ \\
\hline
\end{tabular}

Fuente: elaboración propia a partir de los datos de Auquilla, Astorga y Jiménez (2006)

Con respecto a la calidad del agua de las quebradas ubicadas en la sub cuenca, Auquilla, Astorga y Jiménez (2006) analizaron 8 micro cuencas y evaluaron la calidad por medio de análisis físicoquímicos y bacteriológicos y una técnica de monitoreo biológico. Las muestras las tomaron en 3 puntos diferentes de las quebradas: el primero en la naciente de agua, el segundo aguas abajo de una franja protegida (bosque ribereño) con un uso de suelo definido (pastura con árboles y pastura degradada) y el tercero aguas abajo sin presencia de franja ribereña y con predominio de potreros (pastura con árboles, pastura degradada). En dos quebradas se tomó una cuarta muestra para evaluar la contaminación provocada por los establos.

Los análisis físico-químicos realizados fueron: demanda bioquímica de oxígeno (DBO), demanda química de oxígeno (DQO), sólidos suspendidos, sólidos sedimentables, $\mathrm{pH}$, nitratos y fosfatos. 
Para los análisis microbiológicos se midieron las coliformes fecales y totales. El monitoreo biológico analizó la presencia de organismos macro invertebrados bentónicos como indicadores biológicos. Los resultados obtenidos se muestran en el cuadro 2.

Cuadro 2. Parámetros físico-químicos y microbiológicos promedios obtenidos en 8 microcuencas ubicadas en la sub cuenca del río Jabonal, Esparza, Puntarenas

\begin{tabular}{|l|l|l|}
\hline Parámetro & Valor obtenido & Valor permitido \\
\hline DQO & $19,75 \mathrm{mg} / \mathrm{l}$ & $<20 \mathrm{mg} / \mathrm{l}$ \\
\hline Sólidos suspendidos totales & $24 \mathrm{mg} / \mathrm{l}$ & $<10 \mathrm{mg} / \mathrm{l}$ \\
\hline $\mathrm{pH}$ & $7,1-7,7$ & $6,5-8,5$ \\
\hline Nitratos & $<2 \mathrm{~m} / \mathrm{l}$ & $<5 \mathrm{mg} / \mathrm{l}$ \\
\hline Fósforo total & $0,17 \mathrm{mg} / \mathrm{l}$ & $0,1 \mathrm{mg} / \mathrm{l}$ \\
\hline Coliformes fecales & $330-1014 \mathrm{NMP} / 100 \mathrm{ml}$ & $<20 \mathrm{NMP} / 100 \mathrm{ml}$ \\
\hline
\end{tabular}

Fuente: elaboración propia a partir de los datos de Auquilla, Astorga y Jiménez (2006)

El índice de calidad de agua (ICA) obtenido clasifica el agua de calidad aceptable, con tendencia a levemente contaminada. Se observa efecto acumulativo conforme se desciende desde la parte alta y más protegida de las quebradas hacia la parte baja, con mayor presencia del ser humano. Los resultados obtenidos demostraron que los parámetros de calidad de agua que excedieron el nivel crítico para consumo humano fueron la demanda química de oxígeno (DQO), los sólidos suspendidos, el fósforo total y los coliformes fecales.

- Fuentes de contaminación del agua

Auquilla, Astorga y Jiménez (2006) demostraron que la calidad del agua de las quebradas disminuye conforme el agua proveniente de la naciente baja por ésta hasta llegar a los puntos de uso que le brinda los habitantes de la zona. De acuerdo con Arroyo, Castro y Mejía (2007) y la observación directa realizada durante esta investigación, se puede decir que las principales fuentes de contaminación de agua son provocadas por el inadecuado manejo de aguas residuales domésticas, de residuos animales, de las nacientes y debido al ingreso de ganado a los cuerpos de agua.

Estos resultados concuerdan con la realidad de la región, donde la actividad productiva principal de la zona es el ganado y éste tiene acceso a las quebradas, además las nacientes no tienen una protección adecuada. Igualmente las aguas residuales de muchas casas y establos son vertidas a las quebradas.

Sin embargo a pesar de estos problemas, el ICA obtenido de las quebradas, clasifica el agua de calidad aceptable, con tendencia a levemente contaminada. Situación que podría ser mejorada si se implementan técnicas que ayuden en el tratamiento de las aguas residuales en las fincas y si se protegen las nacientes del ingreso de animales. Labor que ya se ha estado realizando; a partir del 
año 2008, en algunas fincas de estos poblados con la ayuda de la Universidad de Costa Rica y la Asociación Pro cuenca del río Jabonal. Sin embargo, el avance ha sido lento debido a la carencia de recursos económicos.

Residuos

- Cantidad de residuos producidos

De acuerdo con la producción promedio de estiércol para cada especie de animal: $24 \mathrm{~kg} / \mathrm{día}$ el ganado; 3,4 kg/día los cerdos; 100 g/día las gallinas; 16 kg/día los caballos y 1,6 kg/día las cabras (Picado y Añesco, 2005), en estos poblados se generan, aproximadamente, 20620 toneladas de residuos animales por año.

Con respecto a los residuos domésticos, se generan, aproximadamente, 133 toneladas por año. De estos residuos se podría aprovechar el $74,5 \%$, conformado por los residuos reciclables y los residuos orgánicos (Quijada, 2007), o sea 99 toneladas por año.

Todos los entrevistados manifestaron que la cantidad de residuos peligrosos que generan es mínima. El dato exacto no lo conocen. Los residuos peligrosos generados son: galones de herbicidas y frascos de vacunas o medicinas para animales. Cuando están en uso, estos productos son almacenados de tal manera que no presentan un riesgo potencial en la salud humana y el medio ambiente. Las áreas de almacenamiento, por lo general, están ubicadas a distancias considerables de ríos y quebradas y se encuentran bien protegidos esto con el objetivo de evitar accidentes como el derrame del químico.

- Disposición final

Las viviendas no cuentan con el servicio de recolección de los residuos domésticos, lo cual provoca que los habitantes tengan que disponer de estos de la manera que crean más conveniente. El $73 \%$ de las viviendas queman los residuos, el $24 \%$ los entierren y el $3 \%$ los botan a lote baldío. Ver detalle en figura 2. 


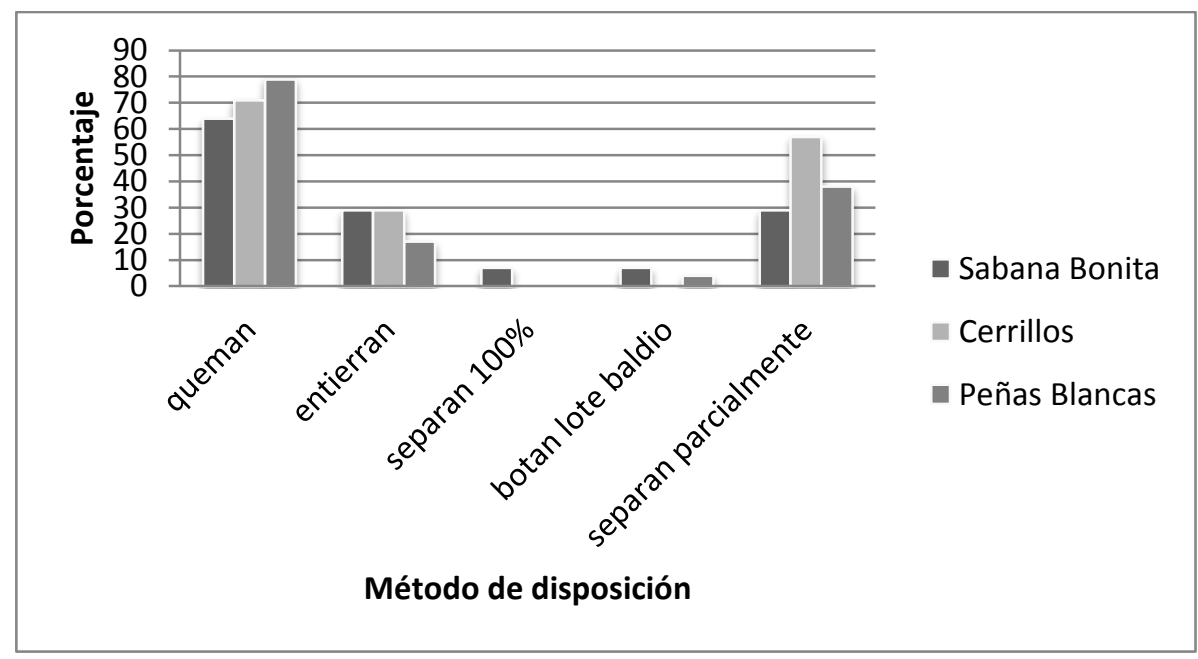

Fuente: elaboración propia

Figura 2. Comparación de los métodos de disposición de los residuos sólidos domésticos utilizados en los poblados de Sabana Bonita, Cerrillos y Peñas Blancas

Sería conveniente realizar un estudio para determinar la composición de los desechos y conocer el potencial de reciclaje. Si el resultado es positivo, se pueden iniciar acciones para el acopio, separación y venta de los residuos. Si es negativo, o sea no tiene potencial de reciclaje, se debe hacer conciencia en la población de utilizar el método que menos afecte el ambiente.

Los residuos generados por los animales, tanto líquidos como sólidos, tampoco son tratados de la mejor manera, la mayoría queda en los potreros o son vertidos en la quebrada más cercana. Existen posibilidades de manejo simples y no costosas, como son el uso de tecnologías limpias, producción de abono y estabulado parcial del ganado. Posibilidad que solo el 7\% (5) de las fincas de zona están utilizando, pero que existe un creciente interés por otros finqueros para implementarlas en sus fincas.

En el $94 \%$ de las viviendas y de las queseras, las aguas residuales grises las vierten directamente al terreno. Esto ocasiona malos olores y en algunos casos contaminación directa de quebradas. Esta situación al igual, que el tratamiento de los residuos animales tiene una fácil solución, por medio de la concientización, capacitación e implementación de tecnologías simples. Es un proceso en el cual la Asociación Pro Cuenca Río Jabonal está trabajando pero de manera lenta debido a la carencia de recursos económicos.

El $100 \%$ de las aguas residuales negras de las viviendas son vertidas a tanque séptico. Todos los encuestados manifiestan creer que el tanque se encuentra en buenas condiciones porque no les da problemas. Pero esto no excluye el hecho de que los tanques puedan tener algún tipo de fuga que pueda estar ocasionando problemas de contaminación de agua. De acuerdo con Ricardo Sancho, presidente ejecutivo del A y A, en el periodo 2006-2010, el uso de tanques sépticos es 
una amenaza para las aguas subterráneas, cuya calidad se puede comprometer ante eventuales filtraciones de materia fecal (Avalos, 2009).

Con la finalidad de determinar si existe diferencia entre los poblados en el método de disposición final de los residuos sólidos domésticos, se aplicó la prueba de Kruskal-Wallis debido a que los datos no siguen una distribución normal. El p-valor obtenido fue de 0,436 y puesto que este valor es mayor que el nivel de significancia de 0,05 de la prueba, se falla en rechazar la hipótesis nula. Por ello se concluye que el método de disposición de los residuos sólidos domésticos no es significativamente diferente entre los tres poblados.

Sin embargo, como en la figura 2 se observa una diferencia entre poblados en la cantidad de viviendas que separan parte de los residuos para enviar a reciclar, se aplicó la prueba de proporciones. Los resultados obtenidos se muestran en el cuadro 3.

Cuadro 3. Resultados del p-valor obtenido de la comparación de proporciones de la cantidad de viviendas que separan parte de los residuos domésticos para enviarlos a reciclar entre los poblados de Sabana Bonita, Cerrillos y Peñas Blancas, Esparza, Puntarenas

\begin{tabular}{|l|c|c|}
\hline Poblado & Sabana Bonita & Peñas Blancas \\
\hline Cerrillos & 0,061 & 0,139 \\
\hline Sabana Bonita & \multicolumn{2}{|c|}{ Fuente: elaboración propia } \\
\hline \multicolumn{3}{|c|}{}
\end{tabular}

Como en todos los casos el p-valor obtenido es mayor que el nivel de significancia de 0,05 de la prueba, se falla en rechazar las hipótesis nulas. Se concluye que no se encontró evidencia estadísticamente significativa entre las proporciones de habitantes de las tres comunidades que separan los residuos domésticos para enviarlos a reciclar.

- Nivel tecnológico

Como se mencionó anteriormente, la eliminación de residuos sólidos, líquidos y peligrosos, es muy básica. En promedio, en el $46 \%$ (32) de las viviendas de los tres poblados se separan algunos residuos sólidos para enviar a reciclar. Esta separación es manual y se traslada en sacos o bolsas a la escuela del poblado, lugar donde la Municipalidad los recoge. Ninguna casa cuenta con recipientes para la separación.

Con respecto al tratamiento de aguas grises, en promedio, solo el 6\% (4) de las casas de los tres poblados cuenta con alguna tecnología implementada para el tratamiento de este tipo de aguas. Dos utilizan un drenaje para filtrar las aguas y dos utilizan humedales artificiales o construidos para el tratamiento de éstas. Para el tratamiento de aguas negras, el $100 \%$ de las casas las dispone en el tanque séptico, no utilizan ninguna tecnología alternativa. 
Para el tratamiento de aguas negras provenientes del lavado de chancheras o establos, solo el $7 \%$ (5) de las fincas utiliza alguna tecnología para el tratamiento de éstas. Dos utilizan biodigestor, por medio del cual producen gas para la cocina, dos utilizan pilas de oxigenación y una construyó un drenaje.

Los residuos peligrosos son enterrados o quemados. No utilizan la metodología de los tres lavados para luego enviar al centro de acopio respectivo.

Únicamente en una finca se produce abono a partir de la boñiga de ganado. Este es procesado por medio de lombricultura.

\section{Capacitación}

Diversas instituciones como el MAG, INA, CATIE y UCR han brindado capacitaciones en temas ambientales en estos poblados. En Sabana Bonita, aproximadamente, el 29\% de las habitantes ha recibido alguna capacitación en la temática ambiental. En Cerrillos el 39\% y en Peñas Blancas el $21 \%$. Algunas de las capacitaciones recibidas son: manejo de residuos sólidos, biodigestores, contaminación de aguas, abono orgánico, uso de suelos, manejo de plaguicidas y mejora de pastos.

Sin embargo, al ser capacitaciones que no siguen ningún plan determinado previamente ni seguimiento, los temas enseñados no se reflejan en el quehacer diario de los habitantes de estos poblados. Como se analiza en Chassoul (2013) la capacitación ambiental ha tenido influencia sobre los conocimientos y actitudes para el manejo de residuos de los habitantes, pero no sobre el comportamiento, porque no todos han implementado lo que se les ha enseñado en esos cursos.

\section{Conservación, recuperación y protección}

En el poblado de Peñas Blancas se encuentra el Refugio de vida silvestre Peñas Blancas. Parte de este refugio se ubica en las afueras de este poblado y otra parte en el cantón de Montes de Oro, Miramar. Abarca un área total de 2400 hectáreas, de las cuales 513,4 hectáreas se ubican en Peñas Blancas. De esta área el $54 \%$ está cubierto por pastos, el $39 \%$ por bosque secundario y el $7 \%$ por vegetación de ribera (Universidad Nacional y Municipalidad de Esparza 2010). Fue creado mediante Ley 7018 artículo 28 del 13 de diciembre de 1985 (Rojas 2011).

A pesar de que este Refugio de vida silvestre, es un área protegida que fue declarada desde el año 1985, no se maneja como tal. En el área no existe la presencia del MINAE y los habitantes tienen libre acceso a ésta, permitiendo la tala de árboles y la cacería. Es un área que podría ser utilizada para turismo rural, actividad que a los vecinos de la zona les gustaría desarrollar, según lo manifestaron en las entrevistas realizadas. 
Relacionado con actividades de conservación, principalmente, el CATIE y la UCR han estado presentes en la zona desde el año 2005 hasta la fecha. EI CATIE estuvo en la zona del año 2005 al 2007 con proyectos silvopastoriles. Estos proyectos fueron aplicados en fincas específicas, por lo cual fueron unas pocas las que se vieron favorecidas. La UCR inició en el 2007 con diversos proyectos enfocados en la gestión participativa e integrada de la sub cuenca. En éstos, la capacitación es abierta al público, pero la aplicación, sobre todo de tecnologías limpias, se realiza finca por finca. Esto ha hecho que los habitantes conozcan de diversas alternativas para el manejo de residuos y la conservación de suelos, pero ha sido un proceso lento debido a los pasos a seguir para la implementación y a la limitación de recursos económicos. Sin embargo, con el tiempo se ha podido observar el cambio de actitud de los habitantes. Al inicio no creían en los beneficios que podían obtener, ahora ya conocen y buscan la implementación de tecnologías limpias en sus fincas. Esto no solo por conciencia ambiental, sino por ahorro o requisitos legales, pero que benefician al ambiente.

\section{Riesgo y vulnerabilidad ambiental}

El mayor riesgo y vulnerabilidad ambiental se encuentra en el poblado de Peñas Blancas. Este poblado está ubicado en una zona de alta susceptibilidad de deslizamiento, el cual se caracteriza por presentar pendientes escarpadas, agrietamiento del terreno, desarrollo de reptación, carcavamiento intenso y evidencias claras de movimientos del terreno, tanto de tipo traslacional como rotacional (Cascante et al 2001).

De acuerdo con Mende y Astorga (2009), el subsuelo de la zona está compuesto por una mezcla de bloques de rocas volcánicas con diámetros muy variables y una matriz limo-arcillosa. Es una mezcla de materiales que ya se ha movido por medio de un flujo de gravedad y en caso de una saturación con agua muestra una muy alta capacidad de moverse impulsado por la fuerza de gravedad. Por la misma razón cualquier tipo de ladera dentro de este tipo de material muestra una estabilidad reducida, especialmente bajo de condiciones climáticas tropicales con alta precipitación. Este movimiento ha ocasionado grietas en la superficie topográfica, daños severos en casas y árboles y postes de luz inclinados. Ver figura 3. 


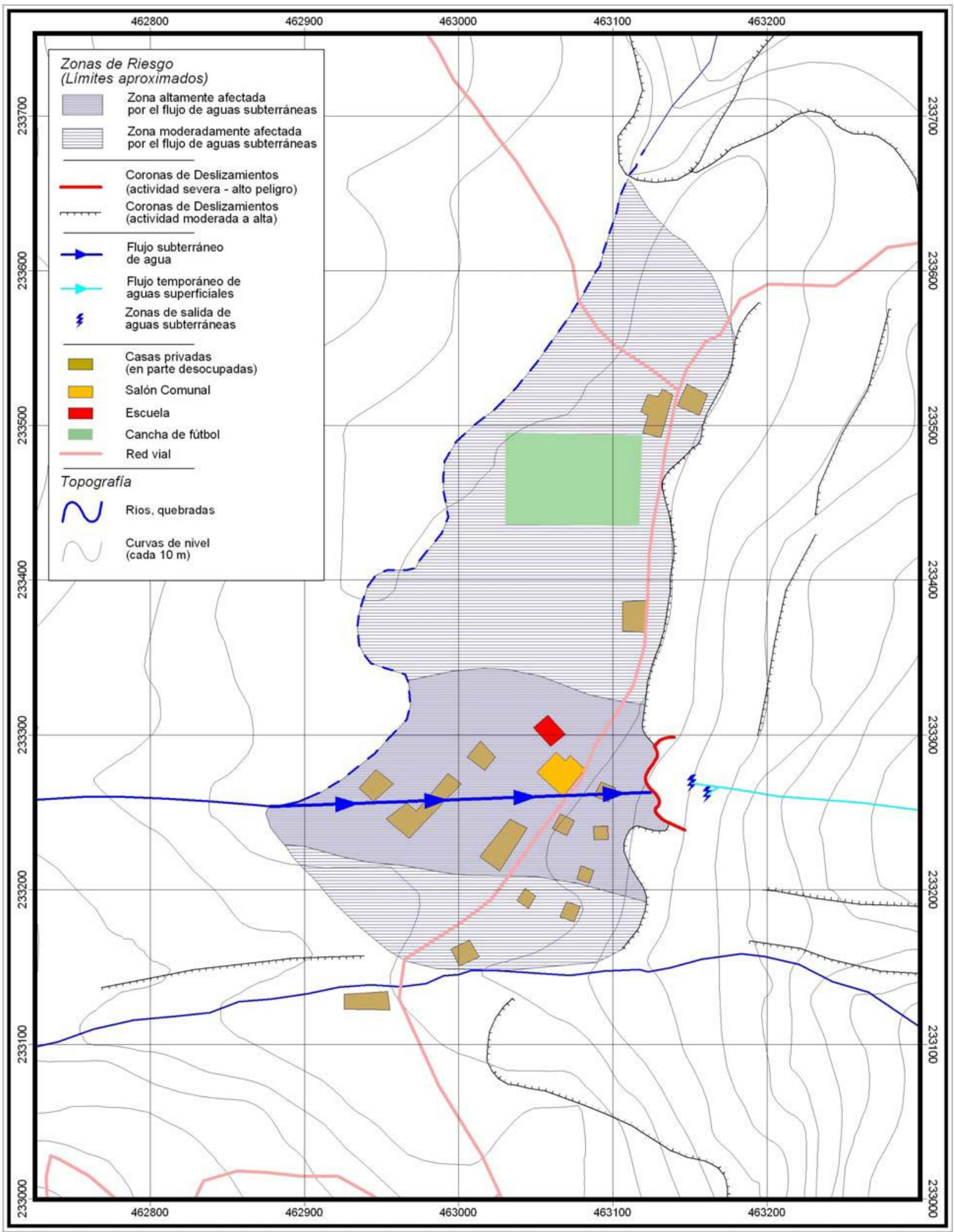

Fuente: Mende y Astorga 2009

Figura 3. Ubicación de las zonas de riesgo en la comunidad de Peñas Blancas, Esparza 
El perfil geológico de la zona de deslizamiento se muestra en la figura 4.

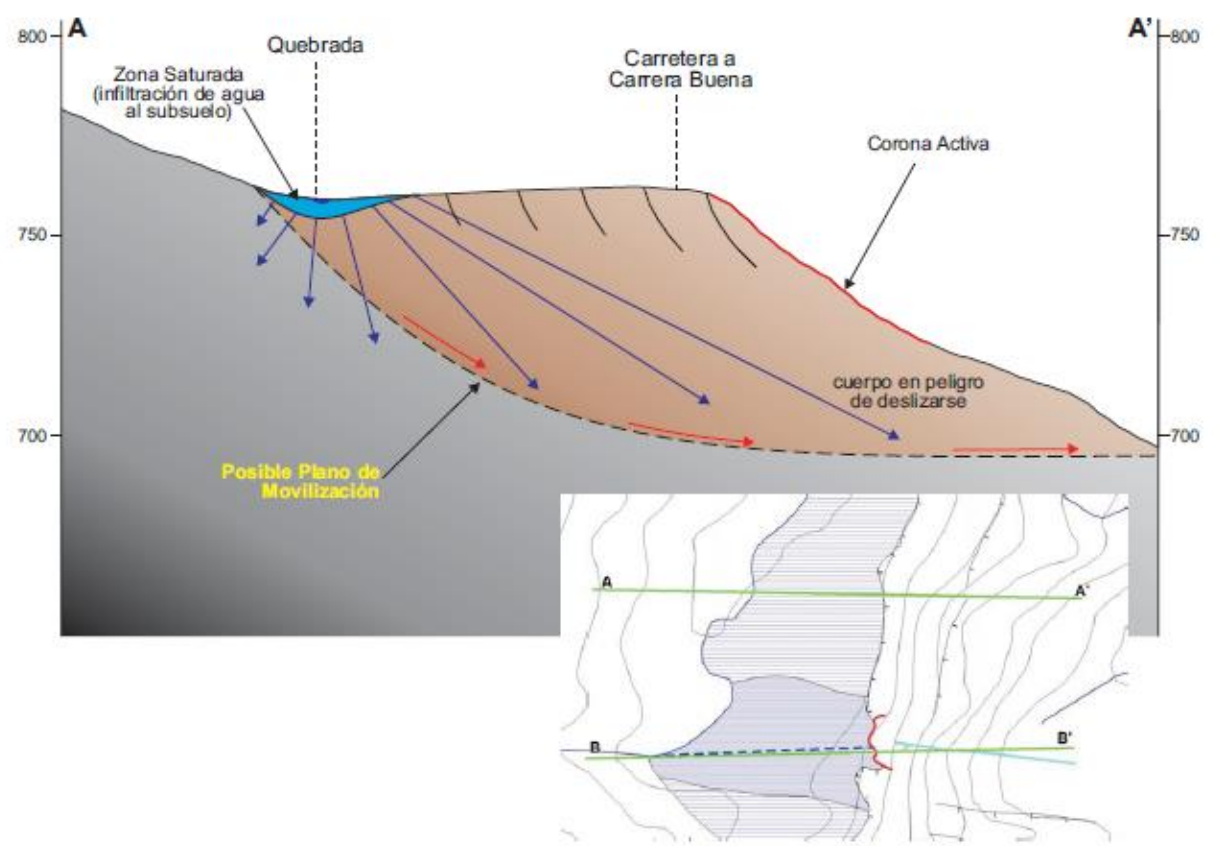

Fuente: Mende y Astorga 2009

Figura 4. Perfil geológicos (A-A' y B-B') sobre la zona en peligro entre la quebrada y el escarpe principal

Mende y Astorga (2009) evaluaron el riesgo por la generación de deslizamientos para la zona entre la quebrada y la corona activa como significativamente alto. Pero establecen que:

no es posible dar un valor exacto para la probabilidad de que la zona en cuestión sea afectada por un evento con el potencial de poner en riesgo serio la población de este sector. Puede ser que esta zona se mantenga relativamente estable durante las próximas decenas de años, por otro lado existe una probabilidad considerable de que sea afectada seriamente por la generación de deslizamientos, especialmente en el caso de condiciones extremos como fuertes tormentas tropicales (Mende y Astorga, 2009, p. 124)

Por su parte, la Comisión Nacional de Emergencias (CNE) en 1995 realizó un informe técnico acerca de la problemática del deslizamiento, denominado "Terrenos propuestos para el proyecto habitacional en distrito San Jerónimo, Peñas Blancas", actualizado en 1999 con el nombre "Información actualizada sobre problemática del deslizamiento Peñas Blancas, cantón de Esparza". Basados en estos informes la Municipalidad de Esparza decidió no otorgar más permisos para la construcción o ubicación de servicios públicos (Cascante et al 2001). Sin embargo, la Municipalidad no toma en cuenta las condiciones económicas de los habitantes. Como ya se analizó, son personas pobres y acostumbradas a vivir en esa zona, por lo cual si se les limita la posibilidad de construcción también les limita las posibilidades de desarrollo. Lo que sería 
convenienste es que la Municipalidad establezca lineamientos para la escogencia del sitio que minimice el riesgo y el tipo de consitrucción permitida. Tal como plantearon Mende y Astorga (2009) no se puede decir exactamente cuando puede ocurrir el deslizamiento.

\section{Análisis general y conclusiones}

Para conocer el equilibrio entre las actividades realizadas por los habitantes y el uso de los recursos naturales, se analizaron variables como energía, suelo, agua, residuos, capacitación ambiental, conservación, recuperación y protección y riesgo y vulnerabilidad ambiental. Los poblados de Cerrillos, Sabana Bonita y Peñas Blancas, presentan problemas ambientales como erosión y compactación de suelos por la ganadería, contaminación de nacientes por mal manejo de éstas, contaminación de quebradas por vertido de aguas residuales de casas y chancheras y mal manejo de residuos.

Con la ayuda del CATIE entre los años 2005 y 2007, se realizaron esfuerzos para introducir el uso de sistemas silvopastoriles y concientizar a los pobladores de la importancia y ventajas de uso de estos sistemas, pero solo unos pocos se vieron beneficiados, motivo por el cual el resto de finqueros siguen con el sistema tradicional. Implementar sistemas silvopastoriles requiere al inicio una fuerte inversión, dinero que los pobladores de la zona no poseen.

En el año 2007, inicia el trabajo en la zona la UCR con el proyecto de gestión integral de la subcuenca. A partir de esta iniciativa se conformó la Asociación Pro Cuenca Río Jabonal, entidad que vela por los problemas ambientales de los 7 poblados ubicados en la sub cuenca.

Debido a la presencia de estas dos instituciones y con el apoyo, en algunas ocasiones del MAG y el INA, la población ha tomado conciencia de los problemas ambientales presentes en la zona. Sin embargo a pesar de que se están realizando acciones para mejorar la situación actual, no existe un plan de acción integral para abordar los problemas detectados e implementar acciones integrales de solución.

Por este motivo, si en la sub cuenca se desea lograr un equilibrio entre el desarrollo económico y la conservación del ambiente, es necesario establecer una estrategia participativa de desarrollo. La cual debe promover el manejo racional de los recursos naturales y que permita mejorar las condiciones sociales y económicas de la población. Esta estrategia podría ser la aplicación de un modelo de desarrollo rural sostenible. 


\section{Referencias bibliográficas}

Arroyo, C.; Castro, J. y Mejía, A. (2007). Metodología de abordaje, identificación y categorización de aspectos ambientales en la subcuenca del río Jabonal y alternativas de solución. Tesis para optar el título de licenciado en Ingeniería Industrial, Sede de Occidente, Universidad de Costa Rica, San Ramón.

Auquilla, R.; Astorga, Y. y Jiménez, F. (2006). Influencia del uso del suelo en la calidad del agua en la subcuenca del río Jabonal, Costa Rica. Recursos Naturales y Ambiente, 48:81-92. Disponible en http://orton.catie.ac.cr/repdoc/A3158E/A3158E.PDF

Avalos, A. (2009). Masivo uso de tanques sépticos amenaza el agua. La Nación, 14 marzo 2009. Obtenido el 29 de abril de 2013 desde http://wvw.nacion.com/ln_ee/2009/marzo/14/pais1905087.html

Cascante, R., Chaves, M., Chinchilla, J., Rodríguez, A. (2001). Estudio geomorfológico y de amenazas naturales para propiciar el plan de ordenamiento territorial de la subcuenca del Río Jabonal, Esparza, Puntarenas. Tesis para optar por el título de licenciado en Geología, Facultad de Ciencias Básicas, Escuela de Geología, Universidad de Costa Rica, San Pedro, M.O.

Chassoul, M. (2013). Análisis del manejo de residuos en Cerrillos, Sabana Bonita y Peñas Blancas, Puntarenas, Costa Rica. Artículo inédito.

Programa Estado de la Nación (2011). Decimoséptimo Informe Estado de la Nación en Desarrollo Humano Sostenible. Programa Estado de la Nación, San José

Mende, A. y Astorga, A. (2009). Informe técnico sobre el estado actual de los deslizamientos activos en los alrededores de la comunidad de Peñas Blancas (Río Jabonal). San José, Costa Rica: Universidad de Costa Rica.

MINAE (2007). Reglamento para la evaluación y clasificación de la calidad de cuerpos de agua superficiales. San José, Costa Rica.

Mora, D y Araya, A. (2008). Estado del agua para consumo humano y saneamiento en costa rica al año 2007. Revista costarricense de salud pública, 32. Recuperado el 7 abril de 2012, de http://www.scielo.sa.cr/scielo.php?pid=S1409-14292008000100004\&script=sci arttext

Picado, J y Añesco, A. (2005). Preparación y uso de abonos orgánicos sólidos y líquidos. Serie agricultura organica № 8. CEDECO (Corporación educativa para el desarrollo costarricense). Recuperado el 26 junio de 2012, de http://www.slideshare.net/unellezvipi/preparacin-y-uso-deabonos-organicos

PROGAI (2006). Memoria del I encuentro-taller manejo sostenible de la cuenca del río Barranca. Cerrillos de Esparza, Febrero, 2006. Universidad de Costa Rica

PROGAI (2007) Informe de labores 2007. Proyecto 802-A7-612. Gestión integrada y participativa de la subcuenca del Rio Jabonal. Universidad de Costa Rica

Programa de las Naciones Unidas para el Desarrollo (2011). Atlas del desarrollo humano cantonal de Costa Rica. San José, Costa Rica: PNUD 
Quijada, O. (2007). Propuesta de un plan de manejo integral de residuos sólidos urbanos para el cantón de Esparza, Puntarenas. Tesis para optar por el grado académico de magister scientiae en Manejo de recursos naturales, Universidad Estatal a Distancia,San José, Costa Rica. Recuperado el 27 de junio, de http://www.uned.ac.cr/investigacion/documents/Tesis_Olman_Quijada.pdf

Rodríguez, D y Urbina, A. Biodigestores ¿Qué son y cómo construirlos?. Programa Regional de Ganadería MAG Grecia. Recuperado el 26 junio de 2012, de http://www.mag.go.cr/bibliotecavirtual/prog-cerdos-biodigestor1.pdf

Rojas, N. (2011) Cuenca río Barranca. Estudio de cuencas hidrográficas de Costa Rica. Instituto Meterológico Nacional. Recuperado el 19 abril de 2012, de http://www.imn.ac.cr

Sepulveda et al. (2002). Metodología para estimar el nivel de desarrollo sostenible en espacios territoriales. San José, Costa Rica: IICA. Disponible en http://www.sidalc.net/repdoc/A5326E/A5326E.PDF

Soto, M. (2012). Asociación Pro cuenca del río Jabonal gana premio conservacionista. Recuperado el 14 de abril de 2013, de http://marcosoto34.wordpress.com/category/ecologia/

Universidad Nacional y Municipalidad de Esparza (2010). Plan regulador del cantón de Esparza: Incorporación de la Variable Ambiental. Esparza, Costa Rica.

Villalobos, M. L y Ramírez, G. R. (2010). Experiencia de gestión integral de cuencas. Subcuenca del río Jabonal, Esparza, Costa Rica. Recuperado el 31 de mayo de 2012, de www.slideshare.net/Ecobook/sub-cuenca-jabonal 